\title{
Consequences of Cosmic Ray Acceleration in Supernova Remnants on their Evolution - the Case of RX J0852.0-4622 (Vela Jr)
}

\section{Bernd Aschenbach ${ }^{1}$}

$P R$ Vaterstetten

Mozartstrasse 8, Vaterstetten, Germany

E-mail: bernd.aschenbachat-online.de

\begin{abstract}
Galactic cosmic rays are generally assumed to be accelerated in supernovae and their remnants. The acceleration of the particles is attributed to their repeatedly interaction with the supernova remnant shock front. Model calculations have shown that a significant fraction of the supernova explosion energy can be converted to energy of cosmic rays. The feedback of this large energy transfer on the dynamical evolution and its impact on the age estimates of supernova remnants is addressed. True ages might be significantly lower than the standard Sedov ages. The impact is described by a quantitative model, which is tested on RX J0852.0-4622 (Vela Jr). Independent of this test conclusions are drawn about the evolution and lifetime of shell-type X-ray synchrotron sources and $\mathrm{TeV}$ gamma-ray sources associated with supernova remnants.
\end{abstract}

Frontier Research in Astrophysics - II

23-28 May 2016

Mondello (Palermo), Italy

${ }^{1}$ Speaker 


\section{Introduction}

Galactic cosmic rays have been suggested to be generated in supernova remnant shock fronts. The energies should reach up to $100 \mathrm{TeV}$ for electrons and up to $10^{15} \mathrm{eV}$ for protons. The observation channels to pin-point an individual source are the observation of hard X-ray synchrotron radiation, i.e., $>1-2 \mathrm{keV}$, and/or of $\mathrm{TeV}$ gamma-rays produced by either the inverse Compton effect of high energy electrons with background photon radiation, and/or the high energy protons accelerated via their SNR shock interaction producing TeV gamma-rays by nuclear collisions with ambient nuclei, predominantly protons.

There are about 294 catalogued galactic SNRs identified as such by their radio emission, about half of them have been detected in X-rays, much less in the optical band. But so far only three SNRs have been found to radiate synchroton $\mathrm{X}$-rays and $\mathrm{TeV}$ gamma-rays from regions confined to their shock fronts. There are other sources with $\mathrm{TeV}$ gamma-ray emission and synchrotron X-rays, like the Crab Nebula, but the emission is from all across the source extent and not confined to the shock front. Therefore these sources are not considered in this study. Three questions arise:

1. why are there so very few objects, if cosmic ray acceleration in SNR shock fronts would be the main source of galactic cosmic rays;

2. is a special type of $\mathrm{SN}$ required for shock acceleration;

3. is the environment around the explosion cloud of importance.

The three SNRs which show X-ray synchrotron emission and $\mathrm{TeV}$ gamma-rays are SN1006 with both thermal and synchrotron X-ray emission and possibly TeV gamma-rays,

RX 1713.7-3946 with synchrotron X-rays, lacking thermal X-rays from the outer shock front completely, and TeV gamma-rays, and RX 0852.0-4622, again without any sign of thermal Xrays but synchrotron $\mathrm{X}$-rays and $\mathrm{TeV}$ gamma-rays. What is special about these three SNRs concerning cosmic ray acceleration in shock fronts?

\section{Creation of cosmic rays by SNR shock acceleration}

The generation of cosmic rays in shocks is treated using the 'theory of non-linear diffusion-like acceleration of particles by their interaction with shock waves', for instance, at the front of shell-type SNRs. Among the results of the application of this theory with respect to SN1006 (Ksenofontov et al., 2005) are firstly, that the energy in cosmic rays can reach 55\% of the total SN explosion energy, secondly, the acceleration time scale can range between just 300 and 700 years, depending weakly on the ambient matter density, and, thirdly the energy increase in cosmic rays saturates after $2000-3000$ years, and the energy is not replenished. In particular, I think, which has not been considered so far, is that the fairly large transfer of energy to cosmic rays should have an effect on the dynamical evolution of the SNR.

As far as I know there is no analytic expression available to describe the time dependence of cosmic ray shock acceleration. Nevertheless, there may be a reliable approximation which is derived using the following seven relations. 


$$
\begin{gathered}
\Delta \mathrm{v}_{\mathrm{cr}} \sim \mathrm{v}_{\mathrm{s}} \\
\Delta \mathrm{v}_{\mathrm{cr}, \mathrm{g}} \sim \mathrm{v}_{\mathrm{s}} \Delta\left(\mathrm{t}^{1 / 2}\right) \\
\mathrm{dE}_{\mathrm{cr}} \sim \mathrm{v}_{\mathrm{s}}^{2} \mathrm{dt} \\
\mathrm{E}_{\mathrm{SN}}=\mathrm{E}_{\mathrm{s}}+\mathrm{E}_{\mathrm{th}}+\mathrm{E}_{\mathrm{kin}}+\mathrm{E}_{\mathrm{cr}} \\
\mathrm{E}_{\mathrm{th}}+\mathrm{E}_{\mathrm{kin}} \sim \mathrm{v}_{\mathrm{s}}^{2} \sim \mathrm{E}_{\mathrm{SN}}-\mathrm{E}_{\mathrm{cr}} \\
\mathrm{dE}_{\mathrm{cr}} \sim-\left(\mathrm{E}_{\mathrm{cr}}-\mathrm{E}_{\mathrm{SN}}\right) \mathrm{dt} \\
\mathrm{E}_{\mathrm{cr}} / \mathrm{E}_{\mathrm{SN}} \sim\left[1-\exp \left(-\mathrm{t} / \mathrm{t}_{\mathrm{cr}}\right)\right]
\end{gathered}
$$

The shock velocity is $\mathrm{v}_{\mathrm{s}} \cdot \Delta \mathrm{v}_{\text {cr }}$ is the increase of the velocity of an ambient proton by crossing the shock which eventually becomes a cosmic ray particle, $t$ is the time, $E_{s}$ is the energy reservoir of the shock, $\mathrm{E}_{\text {th }}$ is the thermal energy and $\mathrm{E}_{\mathrm{kin}}$ is the kinetic energy, respectively, transferred to the ambient matter that passed the shock. Equation 1 illustrates the fundamental process of accelerating ambient particles extracting velocity and energy from the shock and the associated magnetic field for a single crossing, though. Equation 2 is an attempt to describe the process progression, i.e., how the velocity of the suspected cosmic ray particle grows with time, $\Delta \mathrm{v}_{\mathrm{cr}, \mathrm{g}}$. Subsequent crossings of the particle can increase its velocity, but of varying amount, even slowing down its velocity, occasionally. This is why the process is non-linear in time. In fact, it is a diffusion-like process in velocity space. Diffusion-like processes vary with $t^{1 / 2}$. On incremental scale, the result is Eq 2. Eq. 3 is obtained by changing from velocity space to energy space, by squaring $\mathrm{v}_{\mathrm{cr}, \mathrm{g}}, \mathrm{v}_{\mathrm{s}}, \mathrm{t}^{1 / 2}$. Equation 7 shows the resulting relation between total cosmic ray energy and $\mathrm{SN}$ explosion $\mathrm{E}_{\mathrm{SN}}$. It is an exponential with some e-fold time $\mathrm{t}_{\mathrm{cr}}$ which depends on the up-stream magnetic field, its magnitude and morphology, the up-stream matter density, the injection rate of suprathermal particles and their velocity distribution. Comparison with the results of the non-linear theory done for SN1006 by Ksenofontonov et al. (2005) shows that an exponential growth of cosmic ray energy content is not too bad an approximation.

\section{Modification of the Sedov relation}

The general view of the dynamical evolution of SNRs is that the expansion of the explosion of a SN propagating into the ambient environment starts with a phase of free expansion, which at some time, depending on the ambient matter density n, changes to the so-called Sedov-phase, which is energy conservation dominated and during which the expansion velocity of the explosion cloud, i.e., the shock front, slows down with time $t$, indicated by the shock radius $r$ increasing less than proportional to $t$.

$$
\mathrm{r}=\mathrm{const}\left(\mathrm{E}_{\mathrm{SN}} / \mathrm{n}\right)^{1 / 5} \mathrm{t}^{2 / 5}
$$


Measuring the shock velocity and the shock radius the age of the SNR is estimated by $\mathrm{t}_{\text {Sedov }}=$ $2 / 5 \mathrm{r} / \mathrm{v}_{\mathrm{s}}$. This relationship takes care of the energy transfer to kinetic and thermal energy from the shock, which causes the slow-down, but not of the energy losses pumped in cosmic rays. As a working hypothesis I suggest that the original Sedov relation should be modified to take care of the cosmic ray energy consumption. I will use the following relation

$$
\mathrm{E}_{\mathrm{cr}}(\mathrm{t}) / \mathrm{E}_{\mathrm{SN}}=\mathrm{A}\left[1-\exp \left(-\mathrm{t} / \mathrm{t}_{\mathrm{cr}}\right)\right]
$$

such that the original Sedov relation is modified to the following equation 10.

$$
\mathrm{r}=\operatorname{const}\left(\mathrm{E}_{\mathrm{SN}} / \mathrm{n}\right)^{1 / 5} \mathrm{t}^{2 / 5}\left(1-\mathrm{A}\left[1-\exp \left(-\mathrm{t} / \mathrm{t}_{\mathrm{cr}}\right)\right]\right)^{1 / 5}
$$

The immediate effect is that the age of the SNR derived from $r$ and $v_{s}$ changes compared to $t_{\text {Sedov }}$. The ratio of $r / v_{s}$ using equation 10 with $v_{s}$ the time derivative of $r$ shows a single maximum for the parameter $2 \mathrm{r} /\left(5 \mathrm{v}_{\mathrm{s}} \mathrm{t}\right)=\mathrm{t}$ Sedov $/ \mathrm{t}=\mathrm{k}$ for each value of $\mathrm{A}$. This means that the age of the SNR could be lower than the Sedov age by this factor k. For low values of A the reduction in age is negligible, but for higher values of $A$ the age reduction is significant. For example, $\mathrm{A}=0.5, \mathrm{k}=1.16 ; \mathrm{A}=0.7, \mathrm{k}=1.35 ; \mathrm{A}=0.90, \mathrm{k}=2.22 ; \mathrm{A}=0.95, \mathrm{k}=4.2$. The use of the Sedov age as an age estimate becomes fairly unreliable, if $\mathrm{A}$ is approaching values of 0.7 and more. Assuming that this is physically possible, a test object would be, as mentioned in the introduction, RX 0852.0-3946 (Vela Jr).

\section{A test object}

RX 0852.0-3946 (Vela Jr) was discovered during the ROSAT All-Sky-Survey in X-rays (Aschenbach, 1998). A gamma-ray line excess above the background signal due to radioactive ${ }^{44} \mathrm{Ti}$ was found (Iyudin et al., 1998), which, because of its short life-time of 90 years led to the conjecture that this SNR would be young, about 700 years, and because of its X-ray extent should be close-by, maybe as close as 200 pc (Aschenbach et al., 1999). The result of Iyudin et al. concerning the ${ }^{44} \mathrm{Ti}$ signal has not been confirmed by later measurements, the analyses of which, however, are restricted to a point-like source.

Several findings indicate that very unusual celestial events had happened in the $13^{\text {th }}$ century, which might be attributed to a nearby SN. These are, among others, nitrate precipitation markers in Arctic drilled ice cores (Burgess \& Zuber, 2000); sudden increase in atmospheric radiocarbon; the bright star, the Zimbabwe star, probably observed from the Great Zimbabwe monument (Wade, 2015). From Japan, there is a report that on September 12, 1271 an object as bright as the full moon suddenly appeared on the sky about 10 degrees above the horizon, which marks the beginning of the rise of Nichiren Buddhism. All these findings are consistent with the occurrence of a SN in 1271. 
The measured properties of Vela Jr are $\mathrm{r}=1^{\circ} ; \mathrm{v}_{\mathrm{s}}=0.84 \mathrm{arcsec} / \mathrm{yr}$ (Katsuda et al., 2008); $\mathrm{n}<0.029 \mathrm{~d}_{1}{ }^{-1 / 2}$ (Slane et al., 2001); $\mathrm{E}_{\mathrm{cr}}=2.5 \cdot 10^{50} \mathrm{erg} \cdot \mathrm{d}_{1}{ }^{2} / \mathrm{n}$ (Aharonian et al. 2007), if the TeV gamma-ray emission is exclusively attributed to $\pi^{0}$ decay emission. $d_{1}$ is the distance measured in units of $1 \mathrm{kpc}$ and $\mathrm{n}$ is the particle density in $\mathrm{cm}^{-3}$.

The Sedov age determined from the X-ray observation is 1714 years. If the true age would be 730 years ( $\mathrm{SN}$ in 1271) a minimum value of $\mathrm{A}=0.9075$ is required. For $\mathrm{t}$ measured in units of 10000 years, $\mathrm{n}$ in $\mathrm{cm}^{-3}$ and $\mathrm{E}_{\mathrm{SN}}$ in $10^{51} \mathrm{ergs}$, the const in equation 10 is $14 \mathrm{pc}$. Applying the modified Sedov relation (Eq. 10) it turns out that for a canonical value of $\mathrm{E}_{\mathrm{SN}}=10^{51} \mathrm{erg}$, $\mathrm{n}=0.041 \mathrm{~cm}^{-3}$ and the distance to the SNR is $\mathrm{d}=386 \mathrm{pc}$. The cosmic ray e-fold growth time $t_{c r}=338$ years. The values of $n$ and $d$ are fairly insensitive to $t_{c r}$, which could be up to 700 to 800 years for larger values of A without changing the results of $\mathrm{n}$ and d significantly. Reconstructing the velocity time profile shows that the early velocity of the SNR must have been fairly high, i.e. $40000 \mathrm{~km} / \mathrm{s}$ at $\mathrm{t}=20 \mathrm{yrs}$ and $20000 \mathrm{~km} / \mathrm{s}$ at $\mathrm{t}=40 \mathrm{yrs}$. The current, today's, velocity would be $\mathrm{v}_{\mathrm{s}}=1536 \mathrm{~km} / \mathrm{s}$.

Equation 10 implies that $t$ becomes fairly insensitive to the ratio of $r / v_{s}$ for values of $A>0.9$. Allen et al. (2015) have measured the shock velocity and radius at a different rim position of the SNR obtaining an estimated Sedov age 1.7 times larger than the result of the measurements of Katsuda et al. (2008) indicate. Raising the value of $A$ to $A=0.946$ the age of the remnant could be still 730 years. The distance would be $\mathrm{d}=368 \mathrm{pc}$, the density $\mathrm{n}=0.036 \mathrm{~cm}^{-3}$ and $\mathrm{t}_{\mathrm{cr}}=300$ years. In this case the current, today's, velocity would be $\mathrm{v}_{\mathrm{s}}=732 \mathrm{~km} / \mathrm{s}$.

\section{Conclusions}

The three SNRs mentioned, i.e., RX 0852.0-4622, RX 1713.7-3946 and SN1006, appear to have in common a very low ambient density of something $<0.05 \mathrm{~cm}^{-3}$. This suggests that the progenitor star was not likely to be a red supergiant because of the much higher density of the wind embedding such a star at the time of its explosion. This leaves blue supergiants as a possible source or SN type Ia events. This argument is strengthened by the early very high expansion speed, exceeding the generally accepted values of 10000-20000 km/s. SN1987A showed a mean expansion velocity of $40000 \mathrm{~km} / \mathrm{s}$ averaged over the first six years. It appears, that a low ambient matter density and a high explosion velocity, rather than high explosion energy, favor the efficient shock acceleration of particles. That the initial explosion velocity is important is evident from equation 1. The relevance of ambient density $\mathrm{n}$, is obvious from comparing the loss of shock energy to kinetic and thermal energy of the shocked plasma, which increases with $\mathrm{n}$, whereas the cosmic ray energy production increases with $\mathrm{n}^{3 / 16}$ (Ksenofontov et al., 2005). For a value, for instance, of $n=0.06 \mathrm{~cm}^{-3}$ the particle/cosmic ray acceleration efficiency would be 10 times higher in energy transfer than plasma heating. 
Given the values of $t_{c r}$ suggested by the non-linear theory of shock acceleration, and independently in this study, the cosmic ray acceleration starts to saturate after 2000 - 3000 years without any further energy supplied because the shock velocity has become too low (c.f. Eq.1). With no further energy supply and no re-acceleration the cosmic ray particles are subject to radiation losses with the time-scale for electrons being fairly short. If they radiate their energy in an up-stream magnetic field of $5 \mu \mathrm{G}$ the lifetime of the electrons producing $1 \mathrm{keV} \mathrm{X-rays} \mathrm{is}$ 5800 years, and for those producing $10 \mathrm{keV} \mathrm{X}$-rays the lifetime is 1800 years. If the ambient up-stream magnetic field is significantly higher than the typical galactic $5 \mu \mathrm{G}$, the lifetime is shorter, for instance, by a factor of about fifteen for a field strength of $30 \mu \mathrm{G}$. After a couple of a few hundred years the high energy electrons energy are gone and with them the high energy Xray synchrotron radiation and the $\mathrm{TeV}$ gamma-rays if associated with the inverse Compton effect. What is left after at most a couple of a few thousand years is a fairly low surface brightness radio supernova remnant, and possibly a lot of cosmic ray protons, and who knows, isolated pulsars apparently unaccompanied by a supernova remnant.

The combination of low ambient density, high birth shock-velocity and short lifetime of SNR connected cosmic ray electrons might explain the rareness of this special type of SNRs among the SNR population.

\section{References}

[1] L.T. Ksenofontov, E.G. Berezhko, H.J.Völk 2005, Dependence of the gamma-ray emission from SN 1006 on the astronomical parameters, A\&A 443, 973.

[2] B. Aschenbach 1998, Discovery of a young nearby supernova remnant, Nature 396, 141.

[3] A.F. Iyudin, V.Schönfelder, K.Bennett, et al., 1998, Emission from ${ }^{44}$ Ti associated with a previously unknown Galactic supernova covery of a young nearby supernova remnant, Nature 396, 142.

[4] B. Aschenbach, A. Iyudin, V. Schönfelder 1999, Constraints of age, distance and progenitor of the supernova remnant $R X J 0852.0-4622 / G R O J 0852-4642$, A\&A 350, 997.

[5] C.P. Burgess \&K. Zuber, 2000, Footprints of the newly discovered Vela supernova in Antarctic ice cores? Astroparticle Physics 14, 1.

[6] R.P. Wade, 2015, Southern African Cosmogenics and Geomythology of the Great Zimbabwe Cultural Complex since the Mediaeval Trade Network Era.

PhD Thesis. University of Pretoria, http://upetd.uo.ac.za/thesis

[7] S. Katsuda, H. Tsunemi, K. Mori 2008, The Slow X-Ray Expansion of the Northwestern Rim of the Supernova Remnant RX J0852.0-4622, ApJ 278, L35.

[8] P. Slane, J.P. Hughes, R.J. Edgar, et al., 2001, RX J0852.0-4622: Another Nonthermal Shell-Type Supernova Remnant (G266.2-1.2), ApJ 548, 814.

[9] F. Aharonian, A.G. Akhperjanian, A.R. Bazer-Bachi, et al., 2007, H.E.S.S. Observations of the Supernova Remnant RX J0852.0-4622: Shell-Type Morphology and Spectrum of a Widely Extended Very High Energy Gamma-Ray Source, ApJ 661, 236.

[10] G. E. Allen, K. Chow, T. DeLaney et al. 2015, On the Expansion Rate, Age, and Distance of the Supernova Remnant G266.2-1.2 (Vela Jr.), ApJ 798, 12. 


\section{Acknowledgement}

I like to thank Richard Wade for the information about the Zimbabwe event and bringing to my mind the Nichiren event, as well as Franco Giovannelli for further details of the Nichiren event. I also express my thanks to Richard Firestone who communicated the radiocarbon data. 